\title{
Comparison of histological and computed tomographic measurements of pig lung bronchi
}

\author{
Volker H. Schmitt ${ }^{1,2,9}$, Christine Schmitt 3,9 , David Hollemann ${ }^{4}$, \\ Andreas Mamilos ${ }^{5}$, Willi Wagner ${ }^{6,7}$, Oliver Weinheimer ${ }^{6,7,10}$ and \\ Christoph Brochhausen ${ }^{5,8,10}$
}

Affiliations: 'Dept of Cardiology, University Medical Centre, Johannes Gutenberg University of Mainz, Mainz, Germany. ${ }^{2}$ German Center for Cardiovascular Research (DZHK), Partner Site Rhine Main, Mainz, Germany. ${ }^{3}$ Practice Dr Wolf and Colleagues, Mainz, Germany. ${ }^{4}$ Institute of Clinical and Molecular Pathology, State Hospital Horn, Horn, Austria. ${ }^{5}$ REPAIR-lab, Institute of Pathology, University of Regensburg, Regensburg, Germany. ${ }^{6}$ Dept of Diagnostic and Interventional Radiology, University Hospital Heidelberg, Heidelberg, Germany. ${ }^{7}$ Translational Lung Research Centre Heidelberg (TLRC), German Lung Research Centre (DZL), Heidelberg, Germany. ${ }^{8}$ Central Biobank Regensburg, University Regensburg and University Hospital Regensburg, Regensburg, Germany. ${ }^{9}$ Joint first authors. ${ }^{10}$ Joint senior authors.

Correspondence: Christoph Brochhausen, REPAIR-lab, Institute of Pathology, European Institute of Excellence for Tissue Engineering and Regenerative Medicine, University of Regensburg. Franz-Josef-Strauß Allee 11, 93053 Regensburg, Germany. E-mail: christoph.brochhausenđukr.de

\section{ABSTRACT}

Aim: Light microscopy is used as template in the evaluation and further development of medical imaging methods. Tissue shrinkage caused by histological processing is known to influence lung tissue dimensions. In diagnosis of COPD, computed tomography (CT) is widely used for automated airway measurement. The aim of this study was to compare histological and computed tomographic measurements of pig lung bronchi. Methods: Airway measurements of pig lungs were performed after freezing under controlled inflation pressure in a liquid nitrogen bath. The wall thickness of seven bronchi was measured via Micro-CT and CT using the integral-based method (IBM) and the full-width-at-half-maximum method (FWHM) automatically and histologically on frozen and paraffin sections. Statistical analysis was performed using the Wilcoxon test, Pearson's correlation coefficient with a significance level at $\mathrm{p}<0.05$, scatter plots and Bland-Altman plots.

Results: Bronchial wall thickness was smallest in frozen sections (median $0.71 \mathrm{~mm}$ ) followed by paraffin sections (median $0.75 \mathrm{~mm}$ ), Micro-CT (median $0.84 \mathrm{~mm}$ ), and CT measurements using IBM (median $0.68 \mathrm{~mm}$ ) and FWHM (median $1.69 \mathrm{~mm}$ ). Statistically significant differences were found among all tested groups $(\mathrm{p}<0.05)$ except for CT IBM and paraffin and frozen sections and Micro-CT. There was high correlation between all parameters with statistical significance $(\mathrm{p}<0.05)$.

Conclusions: Significant differences in airway measurement were found among the different methods. The absolute measurements with CT IBM were closest to the histological results followed by Micro-CT, whereas CT FWHM demonstrated a distinct divergence from the other groups.

@ERSpublications

Automated measurement techniques advance diagnosis of lung diseases. Pig bronchi wall size varies between Micro-CT, CT IBM, CT FWHM and histology. CT IBM is closest to histological results, followed by Micro-CT. CT FWHM differs highly from all other groups. https://bit.ly/3iRXSrv

Cite this article as: Schmitt VH, Schmitt C, Hollemann D, et al. Comparison of histological and computed tomographic measurements of pig lung bronchi. ERJ Open Res 2020; 6: 00500-2020 [https://doi.org/10.1183/23120541.00500-2020].

This article has supplementary material available from openres.ersjournals.com

Received: 15 July 2020 | Accepted after revision: 14 Sept 2020

Copyright $\odot$ ERS 2020. This article is open access and distributed under the terms of the Creative Commons Attribution Non-Commercial Licence 4.0. 


\section{Introduction}

Imaging procedures such as sonography, radiography, computed tomography (CT) and magnetic resonance imaging are central to diagnostics and play a crucial role in clinical science. In the evaluation of imaging techniques, comparison to light micrographs of histological slides is a commonly used method and is crucial to assessing the genuineness of the medical image compared to the original object in vivo [1-3]. In the development of ultrasonic carotid artery wall thickness measurement and its implementation clinically, the accuracy and interpretation of sonographic measurements gathered in epidemiological studies and clinical trials were verified by correlating ultrasound data with histological measurements obtained from cadavers in vitro and in situ $[1,2]$. Interestingly, the histological specimens in these studies were already corrected for shrinkage due to histological processing $[1,2]$. In CT the ability to size structures correctly plays a decisive role. A good example is that of CT evaluation of renal masses [4], where appropriate sizing is a matter of discussion since studies exist showing a discrepancy between radiological and pathological measurements with overestimation of tumour size in CT measurement [5]. This has implications for preoperative patient management regarding prognosis [6], and in renal surgery, discrepancies in measuring methods can even affect decisions on nephron-sparing surgery in certain patients [7].

In thoracic radiology, CT technology has become indispensable [8]. The automised assessment of airway geometry derived from high-resolution CT scans in particular is of great importance in diagnostics and surveillance of pulmonary diseases [9]. Since various lung diseases are accompanied by an increase in airway wall thickness due to airway remodelling [10-12], based on changes in the airway structure including subepithelial fibrosis, increased smooth muscle mass, submucosal gland enlargement, revascularisation and epithelial alterations [13], qualitative and quantitative analyses of CT images of the lungs are becoming increasingly important, not only for better understanding of lung disease mechanisms and drug targeting strategies, but also in the medical and surgical management of these patients [14-16]. Kosciuch et al. [11] demonstrated a significant correlation between small airway wall thickness and lung function measured via spirometry in patients with bronchial asthma and COPD. This group found increased air trapping in asthma to be reflected in thickening of the airway wall and elevated airway resistance and responsiveness as a result of airway thickening in COPD [11]. Thus, asthma and COPD can be differentiated via airway dimension measurement in high-resolution CT, since the bronchial walls in asthmatic patients are thicker than in those with COPD, whereas airway diameter in asthma seems to be smaller than in COPD [17]. In both diseases, the airway wall thickness and lumen diameter are related to disease severity. The airway wall thickness measured in high-resolution CT images was shown to correlate with functional parameters of airflow obstruction in COPD patients. Moreover, it was demonstrated that the bronchial wall thickness in smokers was significantly greater than that in nonsmokers [18]. In COPD the bronchial dimensions were shown to depend on the smoking status, and smoking-induced airway remodelling was partially reversible after smoking cessation even in long-term heavy smokers [19]. Further, it was demonstrated that short-time airway geometry changes can be detected in severe COPD with quantitative CT [20]. Therefore, huge efforts have been made to develop radiological measurement methods using CT, such as noninvasive $3 \mathrm{D}$ measurements and quantifications of airway geometry and lung parenchyma aiming to advance diagnostic and prognostic methodologies for diseases such as asthma, emphysema, COPD and cystic fibrosis [10-12, 21-24]. Several high-resolution techniques exist such as synchrotron radiation-based CT, which allows the in vivo study of regional lung ventilation in mice and high-resolution lung imaging in humans [25], as well as airway function measurements by direct quantification of stable Xenon gas added as an inhaled contrast agent using K-edge subtraction imaging. The dynamics of Xenon wash-in can then be used to calculate quantitative maps of regional specific lung ventilation [16]. Spiral-CT opens up the possibility of acquiring 3D images of the pulmonary bronchial tree and gives access to quantitative measurements of regional lung volume, ventilation and mechanical properties [16]. Micro-CT represents a promising technique in non-destructive 3D imaging and morphometric analysis with almost microscopic resolution and sufficient soft tissue contrast and presents further possibilities in the assessment of lung micro-architecture [26]. However, conventional methods rely on a large grey value contrast between lung and surrounding tissues and fail on scans with lungs that contain dense pathologies [27]. Also, accurate determination of inner and outer airway wall surfaces of a complete 3D tree structure is challenging because of its complex nature [9]. Moreover, measurement of the density and thickness of thin structures such as airway walls is difficult due to the limited spatial resolution of clinical CT scanners [10]. Therefore, different CT measurement techniques exist such as the full-width-at-half-maximum method (FWHM) and the more advanced integral-based method (IBM). FWHM is thought to overestimate the thickness of bronchus walls, so IBM was developed to obtain a more accurate assessment of anatomical size [10].

In the further development and evaluation of lung imaging methods, comparison of radiological images and radiological measurements of the airway with histological slides is a necessary tool. Alteration of tissue 
volume due to the fixation and histological process is a well-known phenomenon in surgical pathology [28-30] and has also been described in lung tissue [31-40]. As a consequence, it is crucial to investigate the histological correlate to which these procedures are compared, with the aim of identifying the histological procedure that reflects the in vivo structure most accurately. Hence, it is necessary to assess the alteration in volume produced by fixation and processing in a given tissue type, which can then be used as a template for imaging procedures. Ideally, the histological method that best reflects the in vivo situation should be assessed for each tissue. In the present study, for the first time, measurements of the bronchial wall thickness were compared between paraffin and frozen sections as well as between Micro-CT and the two CT methods, IBM and FWHM.

\section{Materials and methods}

The present study was performed using four pig lungs. Since two of these were taken from another animal study and the two remaining lungs were obtained from the local abattoir immediately after slaughtering, no animals were killed for the sole purpose of this study. The experimental method used in this investigation has already been described in detail elsewhere [40, 41]. In brief, four excised pig lungs were intubated using a conventional tracheal tube (Willy Rüsch $\mathrm{GmbH}$, Kernen, Germany) and frozen in liquid nitrogen (temperature $-183^{\circ} \mathrm{C}$ ) under ventilated conditions when visually inflated to total lung capacity by use of a mechanical ventilator (SV 900; Siemens-Elema AB, Sweden) with a positive end-expiratory pressure set at $20 \mathrm{mmHg}$. The frozen lungs were placed in a box which was partly filled with curd to optimise stabilisation for later cutting and was placed in the liquid nitrogen bath again. This compound was scanned with a routine chest CT examination protocol (Brilliance 16; Philips Medical Solutions, Leiden, Netherlands: tube voltage $-120 \mathrm{kV}$, tube current $-120 \mathrm{~mA}$, slice thickness $-1.0 \mathrm{~mm}$, increment $0.8 \mathrm{~mm}$, kernel D). The clinical CT scanner used underwent dedicated routine calibration for water every 3 months and for air daily; the Micro-CT scanner was calibrated to the Hounsfield scale routinely. Immediately after CT image reconstruction, optimal localisations where bronchi crossed the image plane perpendicularly were marked with ink and wire with the help of the CT gantry's laser light. Guided by these marks, the boxes were cut using an anatomical saw (Selekta 3; Mado, Dornhan/Blackforest, Germany), and slices with corresponding bronchi were selected based on macroscopic and CT image examination. Small cuboids containing the selected bronchi were prepared. These cuboids were scanned with a desktop cone-beam Micro-CT scanner ( $\mu$ CT 40; SCANCO Medical, Wangen-Brüttisellen, Switzerland) with an isotropic spacing of $0.02 \mathrm{~mm}$. Then, frozen sections of $5 \mu \mathrm{m}$ thickness were cut from these cuboids using a cryomicrotome and stained with haematoxylin and eosin (H\&E) according to standard protocols. The surface of the cutting edge of the the remaining tissue was marked, and the specimens were fixed in $4 \%$ formalin for at least $12 \mathrm{~h}$ under room temperature. Then, paraffin sections of exactly the same cutting edge as the frozen sections were taken and stained with H\&E. Specimens were excluded once the bronchi could not be microscopically and computertomographically correlated or where intact anatomical structures were missing, e.g. lack of entire intact bronchial wall including the epithelium. Consequently, only perfectly preserved bronchus specimens were used. After this rigorous selection procedure, a total of seven bronchi remained for further evaluation (figure 1). Histological investigation and measurements were performed using a light microscope (BX45; Olympus, Hamburg, Germany) and microscope software (Cell Sense Entry; Olympus, Hamburg, Germany). The optical system of the microscope was calibrated annually by the manufacturer as routine. Digital images were taken with a microscope camera (Olympus SC30; Olympus, Hamburg, Germany). One frozen and one paraffin section were analysed for the selected bronchi, and the wall thickness of the bronchus was investigated using high-power fields (hpf) at 400-fold magnification. For every bronchus 40 measurements were taken at different positions of the bronchus wall in both the paraffin and frozen sections, as previously described [40]. The corresponding CT measurements were performed using the scientific software system YACTA (yet another CT analyser, version 1.1.4.1), as described in detail previously [10]. The greyscale profile across the airway wall was detected by 256 centrifugal rays. The IBM was used to quantify the median airway wall thickness. The wall thickness was determined in areas where the bronchus was surrounded by parenchyma. Localisations that could not be reasonably assessed by the greyscale profile, e.g. when the airway wall was attended by bronchial arteries, were automatically excluded. Data collection was carried out using Microsoft Excel (Microsoft Excel 2010; Microsoft Corporation, Redmond, WA, USA), and statistical analyses were conducted with SPSS Statistics 20 (IBM Deutschland GmbH, Ehningen, Germany). Wilcoxon test and Pearson's correlation coefficient were performed with a significance level at 0.05 , and scatter plots together with Bland-Altman plots were conducted.

\section{Results}

The thickness of the pig bronchus walls was smallest when measured by CT using IBM (median $0.68 \mathrm{~mm}$, mean $0.72 \mathrm{~mm}$, minimum of $0.43 \mathrm{~mm}$, maximum of $0.9 \mathrm{~mm}$ ) followed by measurement in frozen sections (median $0.71 \mathrm{~mm}$, mean $0.66 \mathrm{~mm}$, minimum $0.42 \mathrm{~mm}$, maximum of $0.83 \mathrm{~mm}$ ) then paraffin sections 

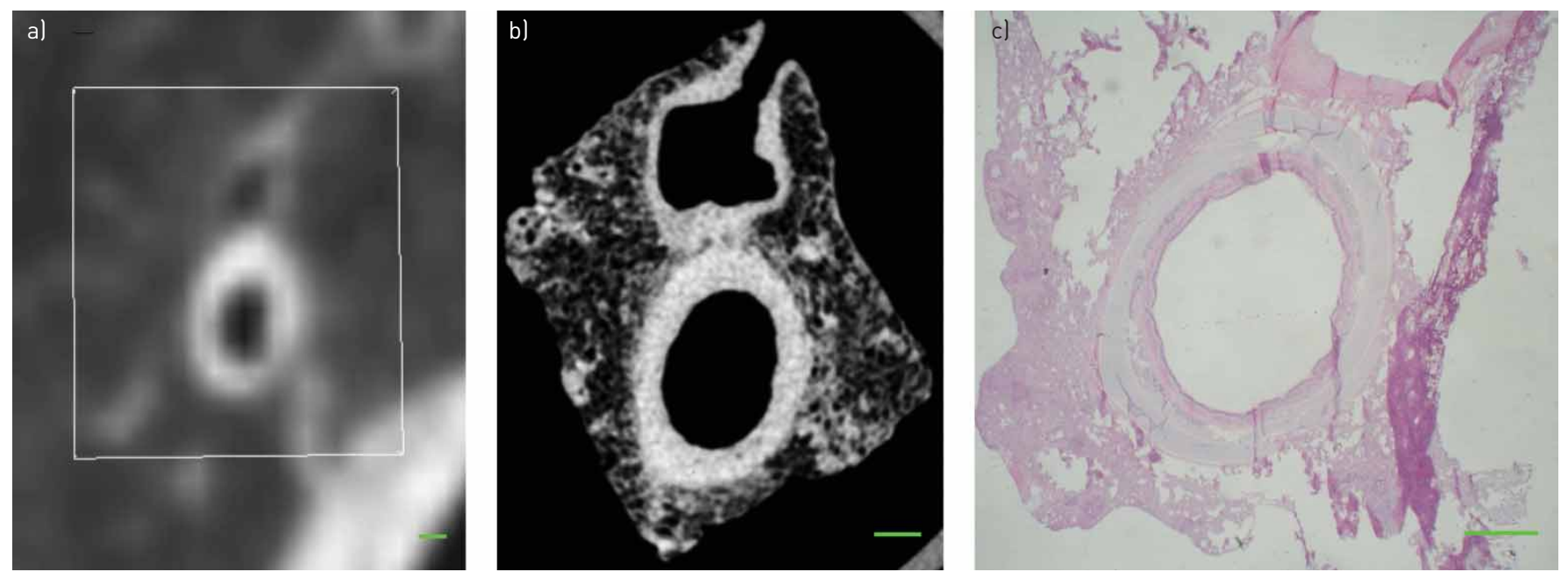

FIGURE 1 Depiction of the same bronchus with different imaging methods. Clear differences in resolution are present between a) computed tomography (CT) and b) MicroCT images. c) Detailed tissue illustration such as distinguishing between cartilage clasps or identifying ciliated epithelium is possible with light microscopy of histological sections only (haematoxylin and eosin staining). CT pixel spacing $0.68 \mathrm{~mm}$, MicroCT pixel spacing $0.02 \mathrm{~mm}$, histological section 20 -fold magnitude. Scale bars=1 $\mathrm{mm}$.

(median $0.75 \mathrm{~mm}$, mean $0.75 \mathrm{~mm}$, minimum $0.05 \mathrm{~mm}$, maximum $0.91 \mathrm{~mm}$ ). The median bronchial wall thickness measured by Micro-CT was $0.84 \mathrm{~mm}$ (mean $0.82 \mathrm{~mm}$, minimum $0.56 \mathrm{~mm}$, maximum $0.95 \mathrm{~mm}$ ). CT measurement using FWHM resulted in the greatest wall thickness (median $1.69 \mathrm{~mm}$, mean $1.64 \mathrm{~mm}$, minimum $1.36 \mathrm{~mm}$, maximum $1.77 \mathrm{~mm}$ ). The results are summarised in table 1 and figure 2 . A Wilcoxon test with a significance level at $\mathrm{p}<0.05$ revealed statistical significance for all groups except for CT using IBM and paraffin sections $(\mathrm{p}=0.237)$, frozen sections $(\mathrm{p}=0.176)$ and Micro-CT $(\mathrm{p}=0.063)$ (supplementary table S1) [40].

Pearson's correlation coefficient revealed high correlation between all groups and statistical significance was present in all of them. The highest correlation was found between Micro-CT and CT using FWHM $(\mathrm{R}=0.929 ; 95 \%$ CI $0.046-0.994, \mathrm{p}=0.003)$, followed by paraffin and frozen sections $(\mathrm{R}=0.902 ; 95 \% \mathrm{CI}$ $0.638-0.988, \mathrm{p}=0.005)$ as well as paraffin sections and Micro-CT $(\mathrm{R}=0.902$; 95\% CI 0.309-0.997, $\mathrm{p}=0.005)$. High correlation was also present between Micro-CT and CT using IBM ( $\mathrm{R}=0.898$; 95\% CI 0.721-0.988, $\mathrm{p}=0.006)$, frozen sections and CT using FWHM ( $\mathrm{R}=0.866$; 95\% CI 0.240-0.981, $\mathrm{p}=0.012)$, paraffin sections and CT using IBM $(\mathrm{R}=0.860 ; 95 \%$ CI $0.291-0.995, \mathrm{p}=0.013)$, frozen sections and Micro-CT $(\mathrm{R}=0.813 ; 95 \%$ CI $0.169-0.994, p=0.026)$, paraffin sections and CT using FWHM ( $\mathrm{R}=0.811 ; 95 \%$ CI $-0.243-0.989$, $\mathrm{p}=0.027)$ as well as frozen sections and CT using IBM ( $\mathrm{R}=0.805$; 95\% CI 0.146-0.987, $\mathrm{p}=0.029)$. The smallest correlation, which was still high, was present between CT using IBM and CT using FWHM $(\mathrm{R}=0.794 ; 95 \%$ CI $-0.151-0.991, \mathrm{p}=0.033)$ (table 2 and supplementary table $\mathrm{S} 2)$. These results are illustrated by regression plots in figures 3 and 4 and supplementary figure S1.

Mean differences and limits of agreement in accordance with the approach of Bland and Altman were calculated between all groups. The smallest mean difference was found to be $0.034 \mathrm{~mm}$ between CT IBM and the paraffin sections, the agreement limits ranging from $-0.148 \mathrm{~mm}$ to $0.217 \mathrm{~mm}$. The largest mean

TABLE 1 Results of the bronchial wall measurements of frozen and paraffin sections as well as Micro computed tomography (Micro-CT) and CT measurements

\begin{tabular}{|c|c|c|c|c|c|}
\hline & Paraffin sections & Frozen sections & Micro-CT & CT IBM & CT FWHM \\
\hline Evaluated bronchi & 7 & 7 & 7 & 7 & 7 \\
\hline Median & 0.75 & 0.71 & 0.84 & 0.68 & 1.69 \\
\hline Range & $0.05-0.91$ & $0.42-0.83$ & $0.56-0.95$ & $0.43-0.90$ & $1.36-1.77$ \\
\hline Interquartile range & $0.72-0.91$ & $0.55-0.76$ & $0.77-0.90$ & $0.61-0.89$ & $1.59-1.75$ \\
\hline
\end{tabular}

All values are given in the dimensional unit millimetres except for the count of evaluated bronchi. CT: computed tomography; IBM: integral-based method; FWHM: full-width-at-half-maximum method. 


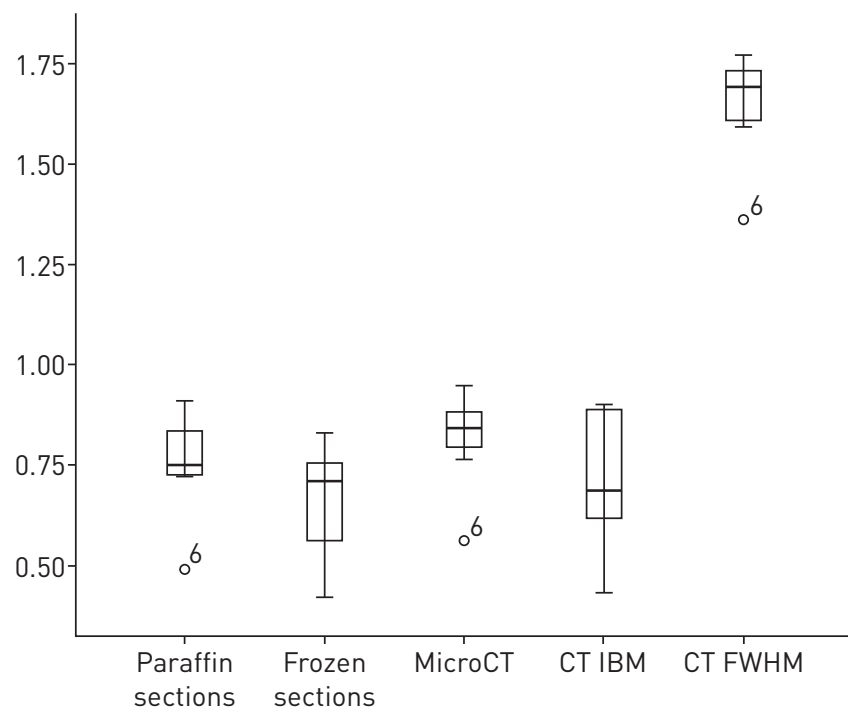

FIGURE 2 Boxplots showing the bronchi wall thickness measurements of frozen and paraffin sections as well as in Micro computed tomography (Micro-CT) and CT measurements using the integral-based method (IBM) and the full-width-at-half-maximum method (FWHM). There was a significant difference between the groups. When histological and computed tomographic measurements were compared, the scoring in paraffin sections and with Micro-CT were closer than the evaluation via frozen section or CT. CT using FWMH revealed a high divergence from the other groups, while CT using IBM was close to the results of the other imaging methods. The measurements on the $y$-axis are in millimetres [40].

difference was found to be $-0.989 \mathrm{~mm}$ between CT FWHM and the frozen sections, the agreement limits ranging from $-1.134 \mathrm{~mm}$ to $-0.844 \mathrm{~mm}$. The Bland-Altman plots are shown in figures 5 and 6 and supplementary figure S1, and the mean differences between all groups are summarised in supplementary table S3.

The results from this study showed that measurements of bronchial walls were close in paraffin and frozen sections, which differed significantly but revealed a high correlation between the two methods (median paraffin sections $0.75 \mathrm{~mm}$, median frozen sections $0.71 \mathrm{~mm}$, divergence of median values $0.04 \mathrm{~mm}$, relative divergence 5\%, Wilcoxon $\mathrm{p}=0.018, \mathrm{R}=0.902, \mathrm{p}=0.005)$. CT using IBM gave measurements that were closest to histological data with a slight underestimation of the bronchial wall thickness compared to paraffin and frozen sections (median CT IBM $0.68 \mathrm{~mm}$, divergence from median paraffin sections $0.07 \mathrm{~mm}$, relative divergence to paraffin sections $9.3 \%$, divergence to median frozen sections $0.03 \mathrm{~mm}$, relative divergence to frozen sections $4.2 \%)$. Wilcoxon test revealed no significant difference ( $\mathrm{p}=0.237$ (paraffin sections), $\mathrm{p}=0.176$ (frozen sections)) to the two histological methods, and simultaneously correlation was high to both groups (paraffin sections and CT IBM: $R=0.860, p=0.013$; frozen sections and CT IBM: $R=0.805$, $\mathrm{p}=0.029$ ). The difference between Micro-CT and histological measurements varied by $12.0 \%$ compared to paraffin sections and by $18.3 \%$ compared to frozen sections (median Micro-CT $0.84 \mathrm{~mm}$, divergence to median paraffin sections $0.09 \mathrm{~mm}$, relative divergence to paraffin sections $12.0 \%$, Wilcoxon $\mathrm{p}=0.043$,

TABLE 2 Heat map showing Pearson's correlation among the investigated imaging modalities

\begin{tabular}{|l|c|c|c|c|c|}
\hline & Paraffin sections & Frozen sections & Micro-CT & CT IBM & CT FWHM \\
\hline Paraffin sections & & 0.902 & 0.902 & 0.86 & 0.811 \\
\hline Frozen sections & 0.902 & & 0.813 & 0.805 & 0.866 \\
\hline Micro-CT & 0.902 & 0.813 & & 0.898 & 0.929 \\
\hline CT IBM & 0.86 & 0.805 & 0.898 & & 0.794 \\
\hline CT FWHM & 0.811 & 0.866 & 0.929 & 0.794 &
\end{tabular}

Highest correlation is given in intense red. Fading colour intensity with declining Pearson's correlation coefficient. CT: computed tomography; IBM: integral-based method; FWHM: full-width-at-half-maximum method. 

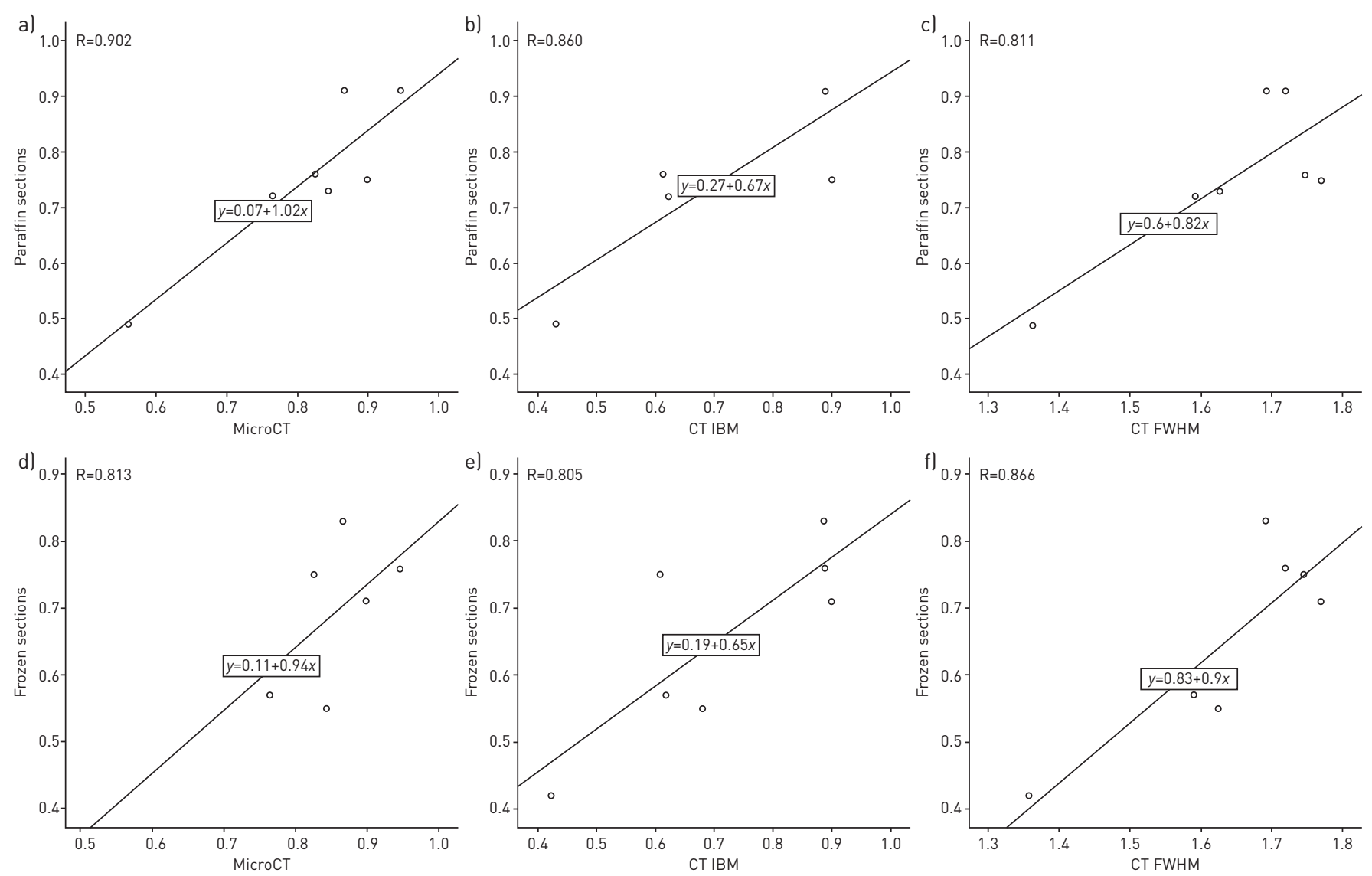

FIGURE 3 Regression plots correlating the two histological methods, a-c) paraffin sections and d-f) frozen sections, with the computed tomographic measurements of a and d) Micro computed tomography (Micro-CT), b and e) CT using the integral-based method (IBM) and c and f) CT using the full-width-at half-maximum method (FWHM). R: Pearson's correlation.

$\mathrm{R}=0.902, \mathrm{p}=0.005$; divergence to median frozen sections $0.13 \mathrm{~mm}$, relative divergence to frozen sections $18.3 \%$, Wilcoxon $\mathrm{p}=0.018, \mathrm{R}=0.813, \mathrm{p}=0.026$ ). Results from CT using FWHM (median $1.69 \mathrm{~mm}$ ) differed considerably from the results of other methods. Compared to histology, there was a divergence of $125.3 \%$ to paraffin sections (divergence to median paraffin sections $0.94 \mathrm{~mm}$, relative divergence $125.3 \%$, Wilcoxon $\mathrm{p}=0.018, \mathrm{R}=0.811, \mathrm{p}=0.027$ ) and $138.0 \%$ to frozen sections (divergence to median frozen sections $0.98 \mathrm{~mm}$, relative divergence $138.0 \%$, Wilcoxon $\mathrm{p}=0.018, \mathrm{R}=0.866, \mathrm{p}=0.012$ ). The difference between $\mathrm{CT}$ using
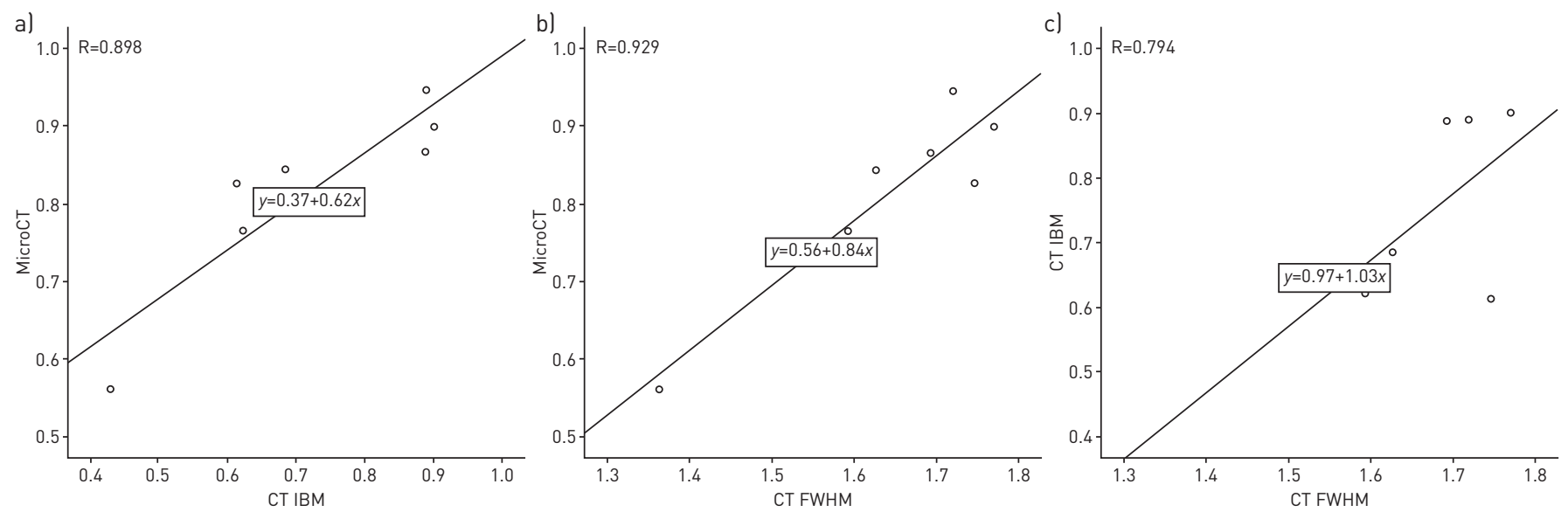

FIGURE 4 Regression plots of Micro computed tomography (Micro-CT), CT using the integral-based method (IBM) and CT using the full-width-at-half-maximum method (FWHM). a) Micro-CT versus CT IBM, b) Micro-CT versus CT FWHM and C) CT IBM versus CT FWHM. R: Pearson's correlation. 

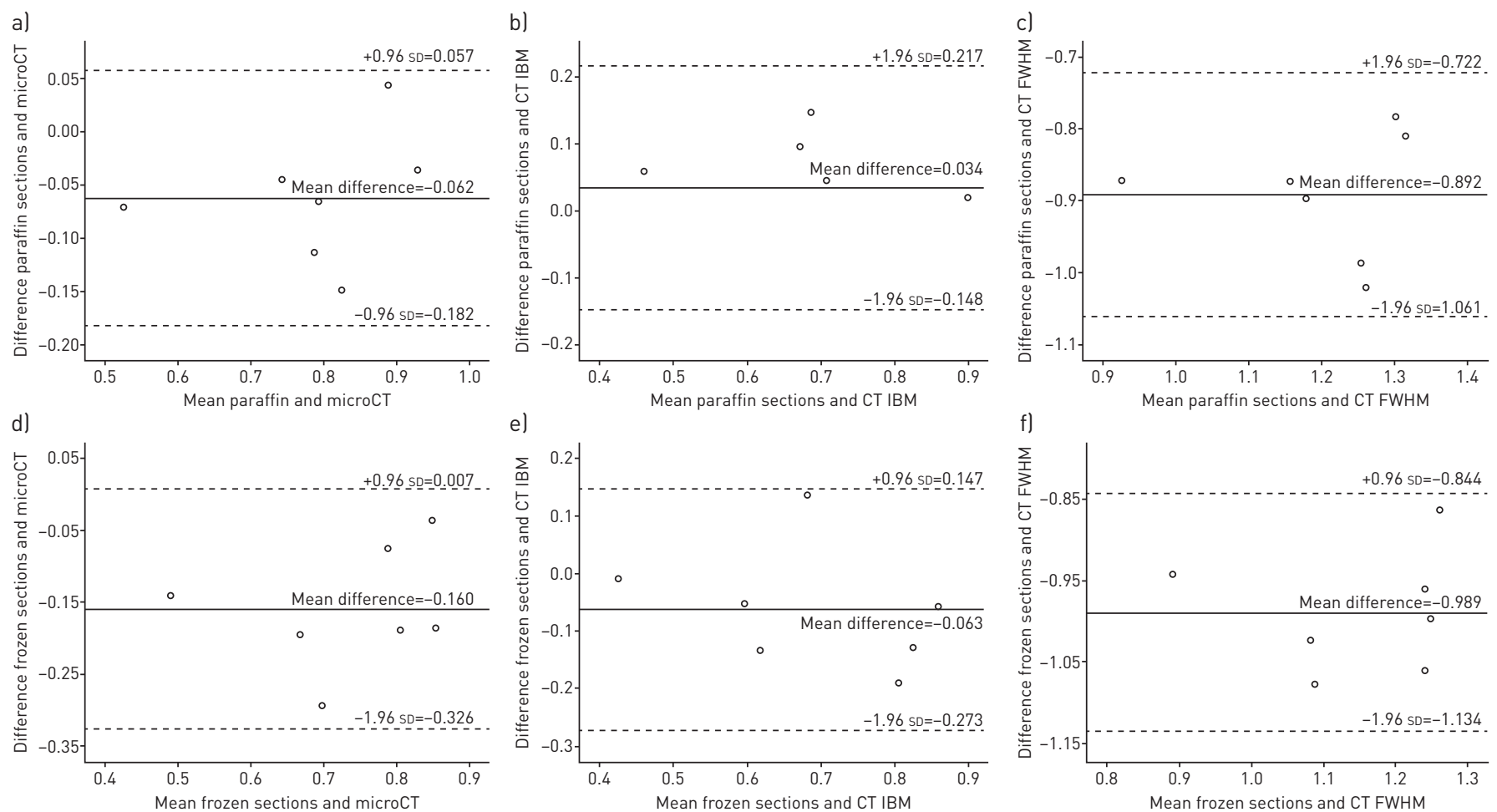

FIGURE 5 Bland-Altman plots showing the distribution between $\mathrm{a}-\mathrm{c}$ ) paraffin sections or $\mathrm{d}-\mathrm{f}$ ) frozen sections with a and d) Micro computed tomography (Micro-CT), b and e) CT using the integral-based method (IBM) and c and f) CT using the full-width-at-half-maximum method (FWHM).

FWHM and Micro-CT was $0.85 \mathrm{~mm}$ (relative divergence $101.2 \%$, Wilcoxon $\mathrm{p}=0.018$ ) with a high correlation of the measurements of both groups $(\mathrm{R}=0.929, \mathrm{p}=0.003)$. The greatest difference among all assessed groups was between the two CT methodologies IBM and FWHM, which was highest overall (median CT IBM $0.68 \mathrm{~mm}$, median CT FWHM $1.69 \mathrm{~mm}$, absolute divergence $1.01 \mathrm{~mm}$, relative divergence $148.5 \%$, Wilcoxon $\mathrm{p}=0.018, \mathrm{R}=0.794, \mathrm{p}=0.033$ ). An overview of absolute and relative median differences between all groups is given in supplementary table S4.

\section{Discussion}

The present study represents the first comparison of different histological and radiological methods, namely paraffin and frozen sections, Micro-CT and CT including the two CT approaches IBM and FWHM, in the quantitative assessment of the airway structure by measuring bronchial wall thickness in the pig. The difference between paraffin and frozen sections amounted to 5\%. CT using IBM produced results that were closest to histological measurements with a difference of $4.2 \%$ compared to frozen and
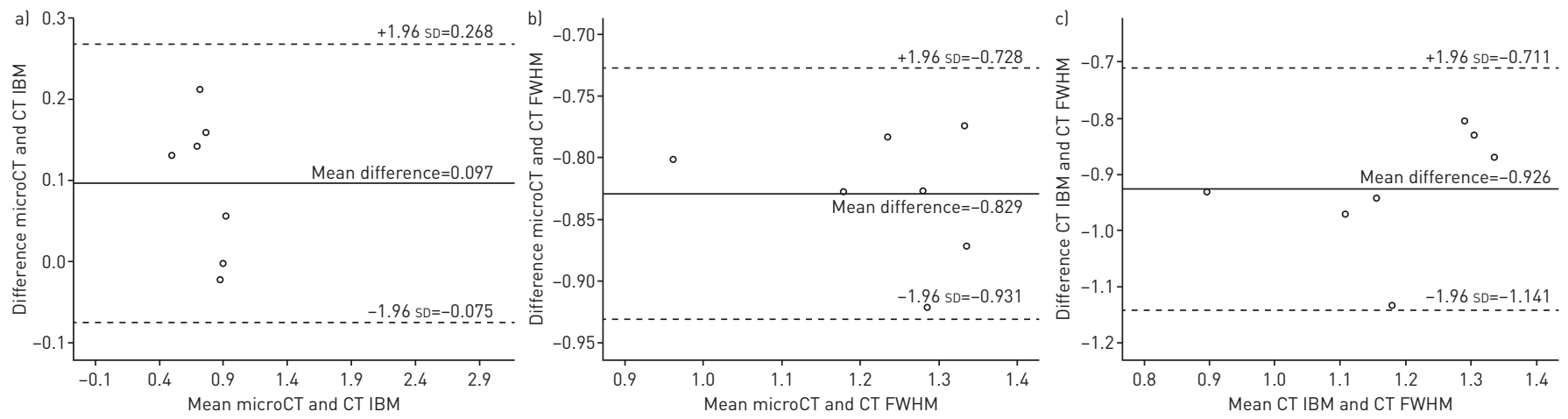

FIGURE 6 Bland-Altman plots showing the distribution between Micro computed tomography (Micro-CT), CT using the integral-based method (IBM) and CT using the full-width-at-half-maximum method (FWHM). a) Micro-CT versus CT IBM, b) Micro-CT versus CT FWHM and C) CT IBM versus CT FWHM. 
9.3\% to paraffin sections. Interestingly, no statistically significant difference but a high and significant correlation was present between CT using IBM and both histological methods. Micro-CT and CT with IBM measurements differed by $19.0 \%$. Interestingly, the two CT methods FWHM and IBM revealed the highest divergence of all groups (148.5\%). While measurements from CT using IBM only differed slightly from histological measurements (9.3\% and 4.2\%, respectively) and Micro-CT (19\%), a large difference was present between all groups and CT using FWHM (divergence of $125.3 \%$ to paraffin and of $138 \%$ to frozen sections, divergence of $101.2 \%$ to Micro-CT). These differences were statistically significant and revealed a high correlation. The difference between Micro-CT and histology was $12.0 \%$ for paraffin sections and $18.3 \%$ for frozen sections.

Assessment of the airway wall by CT offers the advantage of minimal invasiveness and thus has become a useful and reliable tool for airway imaging [13] to enhance understanding and diagnostics of several lung diseases $[11,15,17,18]$. Several methods are currently being tested for accurate quantification of airways and require further development $[9,42]$. To assess and make advancements in imaging techniques it is necessary to compare radiological image data with histological data [43], as illustrated by developments such as carotid artery sonography [1,2] and mammography [44], or even to expose the limitations of radiological methodologies as shown in the assessment of optic nerve invasion of retinoblastoma [45] and the characterisation of adnexal masses [46]. In diagnosing lung diseases such as pulmonary adenocarcinomas, CT represents a useful aid in determining the best surgical method [47] by measurement of tumour size [48]. However, since volume alterations due to histological tissue processing have been observed in lung tissue [31-39], there is a need to investigate such changes. In this context, a comparison of lung adenocarcinoma assessment by CT and gross pathology measurements revealed a statistically significant difference of $18.3 \%$ [49]. Similarly, the results of the present study revealed considerable differences in bronchial wall measurements between histology and CT. These findings are in line with previous investigations in the literature. In a study by IsAKA et al. [50], when the tumour size of lung adenocarcinoma depicted by CT and the measurements performed by the surgical pathologist were compared, tumours measured macroscopically and from paraffin and frozen sections were significantly smaller compared to CT data. In detail, the specimens were macroscopically already significantly smaller compared to the radiological data, but the most considerable loss in size was seen after histological processing. Interestingly, in contrast to the results of the present study concerning the bronchial wall, in the investigation by ISAKA et al. [50] the tissue size in paraffin sections was slightly smaller than in frozen sections. There are many possible reasons for tissue shrinkage in the context of fixation and histological processing, such as exposure to air [30, 51], fixation in alcohol, formalin [52] or formaldehyde [53, 54], freezing [55], embedding in paraffin, cutting and stretching [54]. However, the magnitude of volume loss was shown to vary with tissue type, specimen size, fixative used and tissue processing method [30, 56]. In their study, IsAKA et al. [50] assumed that the difference observed in tumour size between CT and light microscopic methods was due to shrinkage of the lepidic component in the tumour. Regarding the present results, unlike lung adenocarcinoma, the bronchial wall was thinner in frozen than in paraffin sections. This might be caused by the difference in tissue structure of the airway compared to a solid soft tissue tumour like lung adenocarcinoma, which contains considerably more cartilage than soft tissue and may play a role in other shrinkage properties. Apart from evaluating imaging methods in biomedical research, comparison of image features with corresponding histopathology represents the current gold standard for disease assessment [37], and knowledge about fixation and processing-induced tissue shrinkage is crucial in assessing that particular tissue type, especially when samples are compared after different processing procedures [57]. The results of the present study revealed frozen sections and CT using IBM to match best and CT using FWHM to differ most in size from other radiological as well as histological data. However, a review of the literature makes it clear that the methods used in this and former studies might provide different measurements compared to the in situ situation. Therefore, the impact of these differences in size between paraffin and frozen sections has to be investigated further to assess which histological method best represents the in vivo size of the bronchial wall. It is known that the extent of tissue shrinkage depends on several processing parameters such as fixation agent and concentration, and this is why results should be interpreted in the context of the processing method. Knowledge about volume alteration due to fixation and processing together with the further development and improvement of medical imaging methods is crucial since differences between radiological and pathological tissue volumes could have considerable implications in the treatment and prognosis of patients [49]. Furthermore, tissue shrinkage due to fixation, embedding and staining in the lung has been shown to vary considerably among mammalian species revealing the need for interspecies studies and comparability [31]. The present study reveals crucial results for histological and radiological airway measurement, especially in the context of further development of radiological methods.

Acknowledgements: We thank Silke Mitschke from the Institute of Pathology, University Medical Center of the Johannes Gutenberg University of Mainz, Mainz, Germany for the excellent technical support. We also thank Hermann 
Götz (Dept for Oral and Maxillofacial Surgery, University Medical Centre Mainz, Germany) for his technical support in dealing with the Micro-CT scanner. This work contains parts of the MD thesis of C. Schmitt. The present study was performed with four pig lungs. Since two of these were gained from another animal study and the two remaining lungs were obtained from the local abattoir immediately after slaughtering, no animals were killed for the sole purpose of this study.

Conflict of interest: V.H. Schmitt has nothing to disclose. C. Schmitt has nothing to disclose. D. Hollemann has nothing to disclose. A. Mamilos has nothing to disclose. W. Wagner has nothing to disclose. O. Weinheimer reports grants from DFG German Research Foundation during the conduct of the study. C. Brochhausen has nothing to disclose.

Support statement: This study was supported by the German Research Foundation (Deutsche Forschungsgemeinschaft DFG, WE 4691/2-1). Funding information for this article has been deposited with the Crossref Funder Registry.

\section{References}

1 Gamble G, Beaumont B, Smith $\mathrm{H}$, et al. B-mode ultrasound images of the carotid artery wall: correlation of ultrasound with histological measurements. Atherosclerosis 1993; 102: 163-173.

2 Wong M, Edelstein J, Wollman J, et al. Ultrasonic-pathological comparison of the human arterial wall Verification of intima-media thickness. Arterioscler Thromb 1993; 13: 482-486.

3 Choi SM, Choi DK, Kim TH, et al. A comparison of radiologic tumor volume and pathologic tumor volume in renal cell carcinoma (RCC). PLoS One 2015; 10: e0122019.

4 Alicioglu B, Kaplan M, Yurut-Caloglu V, et al. Radiographic size versus surgical size of renal masses: which is the true size of the tumor? J BUON 2009; 14: 235-238.

5 Lee SE, Lee WK, Kim DS, et al. Comparison of radiographic and pathologic sizes of renal tumors. World J Urol 2010; 28: 263-267.

6 Jeffery NN, Douek N, Guo DY, et al. Discrepancy between radiological and pathological size of renal masses. BMC Urol 2011; 11: 2 .

7 Schlomer B, Figenshau RS, Yan Y, et al. How does the radiographic size of a renal mass compare with the pathologic size? Urology 2006; 68: 292-295.

8 Sluimer I, Schilham A, Prokop M, et al. Computer analysis of computed tomography scans of the lung: a survey. IEEE Trans Med Imaging 2006; 25: 385-405.

9 Xu Z, Bagci U, Foster B, et al. Spatially constrained random walk approach for accurate estimation of airway wall surfaces. Med Image Comput Comput Assist Interv 2013; 16: Pt. 2, 559-566.

10 Weinheimer $\mathrm{O}$, Achenbach $\mathrm{T}$, Bletz $\mathrm{C}$, et al. About objective 3-D analysis of airway geometry in computerized tomography. IEEE Trans Med Imaging 2008; 27: 64-74.

11 Kosciuch J, Krenke R, Gorska K, et al. Relationship between airway wall thickness assessed by high-resolution computed tomography and lung function in patients with asthma and chronic obstructive pulmonary disease J Physiol Pharmacol 2009; 60: Suppl. 5, 71-76.

12 Nakano Y, Van Tho N, Yamada H, et al. Radiological approach to asthma and COPD - the role of computed tomography. Allergol Int 2009; 58: 323-331.

13 Górska K, Krenke R, Kosciuch J, et al. Relationship between airway inflammation and remodeling in patients with asthma and chronic obstructive pulmonary disease. Eur J Med Res 2009; 14: Suppl. 4, 90-96.

14 Estépar RSJ, Washko GG, Silverman EK, et al. Accurate airway wall estimation using phase congruency. Med Image Comput Comput Assist Interv 2006; 9: Pt. 2, 125-134.

15 Kosciuch J, Krenke R, Gorska K, et al. Airway dimensions in asthma and COPD in high resolution computed tomography: can we see the difference? Respir Care 2013; 58: 1335-1342.

16 Bayat S, Porra L, Suhonen $\mathrm{H}$, et al. Imaging of lung function using synchrotron radiation computed tomography: what's new? Eur J Radiol 2008; 68: Suppl. 3, S78-S83.

17 Kurashima K, Hoshi T, Takayanagi N, et al. Airway dimensions and pulmonary function in chronic obstructive pulmonary disease and bronchial asthma. Respirology 2012; 17: 79-86.

18 Achenbach T, Weinheimer O, Biedermann A, et al. MDCT assessment of airway wall thickness in COPD patients using a new method: correlations with pulmonary function tests. Eur Radiol 2008; 18: 2731-2738.

19 Jobst BJ, Weinheimer O, Buschulte $\mathrm{T}$, et al. Longitudinal airway remodeling in active and past smokers in a lung cancer screening population. Eur Radiol 2019; 29: 2968-2980.

20 Konietzke P, Wielputz MO, Wagner WL, et al. Quantitative CT detects progression in COPD patients with severe emphysema in a 3-month interval. Eur Radiol 2020; 30: 2502-2512.

21 Rodriguez A, Ranallo FN, Judy PF, et al. CT reconstruction techniques for improved accuracy of lung CT airway measurement. Med Phys 2014; 41: 111911.

22 Revel M-P, Faivre J-B, Remy-Jardin M, et al. Automated lobar quantification of emphysema in patients with severe COPD. Eur Radiol 2008; 18: 2723-2730.

23 Ukil S, Reinhardt JM. Smoothing lung segmentation surfaces in three-dimensional X-ray CT images using anatomic guidance. Acad Radiol 2005; 12: 1502-1511.

24 Wielpütz MO, Eichinger M, Weinheimer $\mathrm{O}$, et al. Automatic airway analysis on multidetector computed tomography in cystic fibrosis: correlation with pulmonary function testing. J Thorac Imaging 2013; 28: 104-113.

25 Wagner WL, Wuennemann F, Pacile S, et al. Towards synchrotron phase-contrast lung imaging in patients - a proof-of-concept study on porcine lungs in a human-scale chest phantom. J Synchrotron Radiat 2018; 25: Pt. 6, $1827-1832$.

26 Langheinrich AC, Bohle RM, Breithecker A, et al. [Micro-computed tomography of the vasculature in parenchymal organs and lung alveoli]. Rofo 2004; 176: 1219-1225.

27 Sluimer I, Prokop M, van Ginneken B. Toward automated segmentation of the pathological lung in CT. IEEE Trans Med Imaging 2005; 24: 1025-1038.

28 West MJ. Tissue shrinkage and stereological studies. Cold Spring Harb Protoc 2013; 2013: pdb.top071860.

29 Salmhofer W, Rieger E, Soyer HP, et al. Influence of skin tension and formalin fixation on sonographic measurement of tumour thickness. J Am Acad Dermatol 1996; 34: 34-39. 

enucleation for retinoblastoma. Arch Ophthalmol 2003; 121: 73-75.

31 Lum H, Mitzner W. Effects of $10 \%$ formalin fixation on fixed lung volume and lung tissue shrinkage. A comparison of eleven laboratory species. Am Rev Respir Dis 1985; 132: 1078-1083.

32 Sutinen S, Pääkko P, Lahti R. Post-mortem inflation, radiography, and fixation of human lungs. A method for radiological and pathological correlations and morphometric studies. Scand J Respir Dis 1979; 60: 29-35.

33 Forrest JB. Measurement of the volume shrinkage of lung tissue due to rapid freezing followed by freeze substitution. J Physiol 1969; 202: 108P+.

34 Carney DE, Bredenberg CE, Schiller HJ, et al. The mechanism of lung volume change during mechanical ventilation. Am J Respir Crit Care Med 1999; 160: 5 Pt. 1, 1697-1702.

35 Tsunoda S, Martin CJ. Lung tissue shrinkage after freeze substitution for histologic study. Am Rev Respir Dis 1973; 107: 876-878.

36 Fukaya H, Martin CJ. Lung tissue shrinkage for histologic preparations. Am Rev Respir Dis 1969; 99: 946-948.

37 Hoang DM, Voura EB, Zhang C, et al. Evaluation of coils for imaging histological slides: signal-to-noise ratio and filling factor. Magn Reson Med 2014; 71: 1932-1943.

38 Thurlbeck WM. Post-mortem lung volumes. Thorax 1979; 34: 735-739.

39 Mazzone RW, Kornblau S, Durand CM. Shrinkage of lung after chemical fixation for analysis of pulmonary structure-function relations. J Appl Physiol Respir Environ Exerc Physiol 1980; 48: 382-385.

40 Schmitt C. Bronchialwandvermessung in der modernen Diagnostik - Vergleich histologischer und bildgebender Verfahren im Tierversuch. Wiesbaden, Germany, Springer Research, 2017.

41 Achenbach T, Weinheimer O, Brochhausen C, et al. Accuracy of automatic airway morphometry in computed tomography - correlation of radiological-pathological findings. Eur J Radiol 2012; 81: 183-188.

42 Achenbach T, Weinheimer O, Dueber C, et al. Influence of pixel size on quantification of airway wall thickness in computed tomography. J Comput Assist Tomogr 2009; 33: 725-730.

43 Achenbach T, Weinheimer O, Buschsieweke C, et al. [Fully automatic detection and quantification of emphysema on thin section MD-CT of the chest by a new and dedicated software]. Rofo 2004; 176: 1409-1415.

44 Tinnemans JG, Wobbes T, Holland R, et al. Mammographic and histopathologic correlation of nonpalpable lesions of the breast and the reliability of frozen section diagnosis. Surg Gynecol Obstet 1987; 165: 523-529.

45 Wilson MW, Rodriguez-Galindo C, Billups C, et al. Lack of correlation between the histologic and magnetic resonance imaging results of optic nerve involvement in eyes primarily enucleated for retinoblastoma. Ophthalmology 2009; 116: 1558-1563.

46 Bazot M, Nassar-Slaba J, Thomassin-Naggara I, et al. MR imaging compared with intraoperative frozen-section examination for the diagnosis of adnexal tumors; correlation with final histology. Eur Radiol 2006; 16: 2687-2699.

47 Saito H, Kameda Y, Masui K, et al. Correlations between thin-section CT findings, histopathological and clinical findings of small pulmonary adenocarcinomas. Lung Cancer 2011; 71: 137-143.

48 Jiang B, Takashima S, Miyake C, et al. Thin-section CT findings in peripheral lung cancer of $3 \mathrm{~cm}$ or smaller: are there any characteristic features for predicting tumor histology or do they depend only on tumor size? Acta Radio 2014; 55: 302-308

49 Lampen-Sachar K, Zhao B, Zheng J, et al. Correlation between tumor measurement on Computed Tomography and resected specimen size in lung adenocarcinomas. Lung Cancer 2012; 75: 332-335.

50 Isaka T, Yokose T, Ito H, et al. Comparison between CT tumor size and pathological tumor size in frozen section examinations of lung adenocarcinoma. Lung Cancer 2014; 85: 40-46.

51 Goldstein NS, Soman A, Sacksner J. Disparate surgical margin lengths of colorectal resection specimens between in vivo and in vitro measurements. The effects of surgical resection and formalin fixation on organ shrinkage. Am J Clin Pathol 1999; 111: 349-351.

52 Noguchi M, Furuya S, Takeuchi T, et al. Modified formalin and methanol fixation methods for molecular biological and morphological analyses. Pathol Int 1997; 47: 685-691.

53 Moelans CB, ter Hoeve N, van Ginkel JW, et al. Formaldehyde substitute fixatives. Analysis of macroscopy morphologic analysis, and immunohistochemical analysis. Am J Clin Pathol 2011; 136: 548-556.

54 Fox CH, Johnson FB, Whiting J, et al. Formaldehyde fixation. J Histochem Cytochem 1985; 33: 845-853.

55 Gardner ES, Sumner WT, Cook JL. Predictable tissue shrinkage during frozen section histopathologic processing for Mohs micrographic surgery. Dermatol Surg 2001; 27: 813-818.

56 Kerns MJJ, Darst MA, Olsen TG, et al. Shrinkage of cutaneous specimens: formalin or other factors involved? J Cutan Pathol 2008; 35: 1093-1096.

57 Rieger J, Twardziok S, Huenigen $\mathrm{H}$, et al. Porcine intestinal mast cells. Evaluation of different fixatives for histochemical staining techniques considering tissue shrinkage. Eur J Histochem 2013; 57: e21. 